\title{
Free-floating adult human brain-derived slice cultures as a model to study the neuronal impact of Alzheimer's disease-associated $A \beta$ oligomers
}

\author{
Niele D. Mendes ${ }^{\mathrm{a}, \mathrm{b}}$, Artur Fernandes ${ }^{\mathrm{a}, \mathrm{c}}$, Glaucia M. Almeida ${ }^{\mathrm{a}}$, Luis E. Santos ${ }^{\mathrm{d}}$, Maria Clara Selles ${ }^{\mathrm{d}}$, \\ N.M. Lyra e Silva ${ }^{\mathrm{d}}$, Carla M. Machado ${ }^{\mathrm{e}}$, José A.C. Horta-Júnior ${ }^{\mathrm{e}}$, Paulo R. Louzada ${ }^{\mathrm{f}}$, \\ Fernanda G. De Felice ${ }^{\mathrm{d}, g}$, Soniza Alves-Leon ${ }^{\mathrm{h}}$, Jorge Marcondes ${ }^{\mathrm{h}}$, João Alberto Assirati Jr. ${ }^{\mathrm{i}}$, \\ Caio M. Matias ${ }^{\mathrm{i}}$, William L. Klein ${ }^{\mathrm{j}}$, Norberto Garcia-Cairasco ${ }^{\mathrm{c}}$, Sergio T. Ferreira ${ }^{\mathrm{d}, \mathrm{k}}$, \\ Luciano Neder ${ }^{\mathrm{b}, \mathrm{l}}$, Adriano Sebollela ${ }^{\mathrm{a}, *}$
}

a Dept. Biochemistry and Immunology, Ribeirao Preto Medical School, University of Sao Paulo, SP, Brazil

${ }^{\mathrm{b}}$ Dept. Pathology and Forensic Medicine, Ribeirao Preto Medical School, University of Sao Paulo, SP, Brazil

${ }^{\mathrm{c}}$ Dept. Physiology, Ribeirão Preto Medical School, University of São Paulo, SP, Brazil

d Institute of Medical Biochemistry, Federal University of Rio de Janeiro, RJ, Brazil

e Department of Anatomy, Institute of Biosciences, São Paulo State University, SP, Brazil

${ }^{\mathrm{f}}$ Institute of Biomedical Sciences, Federal University of Rio de Janeiro, RJ, Brazil

${ }^{g}$ Centre for Neuroscience Studies, Department of Biomedical and Molecular Sciences, Queen's University, Kingston, ON, Canada

${ }^{\mathrm{h}}$ Hospital Universitário Clementino Fraga Filho, Federal University of Rio de Janeiro, RJ, Brazil

${ }^{\mathrm{i}}$ Ribeirão Preto Medical School Clinical Hospital, University of São Paulo, SP, Brazil

${ }^{\mathbf{j}}$ Department of Neurobiology, Northwestern University, IL, USA

${ }^{\mathrm{k}}$ Institute of Biophysics Carlos Chagas Filho, Federal University of Rio de Janeiro, RJ, Brazil

${ }^{1}$ Barretos Cancer Hospital, Barretos, SP, Brazil

A R T I C L E I N F O

\section{Keywords:}

Tissue slices

Organotypic culture

Human brain

Alzheimer's disease

A $\beta$ oligomers

Epilepsy

\begin{abstract}
A B S T R A C T
Background: Slice cultures have been prepared from several organs. With respect to the brain, advantages of slice cultures over dissociated cell cultures include maintenance of the cytoarchitecture and neuronal connectivity. Slice cultures from adult human brain have been reported and constitute a promising method to study neurological diseases. Despite this potential, few studies have characterized in detail cell survival and function along time in short-term, free-floating cultures.

New Method: We used tissue from adult human brain cortex from patients undergoing temporal lobectomy to prepare $200 \mu \mathrm{m}$-thick slices. Along the period in culture, we evaluated neuronal survival, histological modifications, and neurotransmitter release. The toxicity of Alzheimer's-associated A $\beta$ oligomers (A $\beta O s$ ) to cultured slices was also analyzed.

Results: Neurons in human brain slices remain viable and neurochemically active for at least four days in vitro, which allowed detection of binding of A $\beta O$ s. We further found that slices exposed to A $\beta O$ s presented elevated levels of hyperphosphorylated Tau, a hallmark of Alzheimer's disease.

Comparison with Existing Method(s): Although slice cultures from adult human brain have been previously prepared, this is the first report to analyze cell viability and neuronal activity in short-term free-floating cultures as a function of days in vitro.

Conclusions: Once surgical tissue is available, the current protocol is easy to perform and produces functional slices from adult human brain. These slice cultures may represent a preferred model for translational studies of neurodegenerative disorders when long term culturing in not required, as in investigations on $\mathrm{A} \beta \mathrm{O}$ neurotoxicity.
\end{abstract}

\section{Introduction}

The sporadic form of Alzheimer's Disease (AD) accounts for more than 95\% of AD cases worldwide (Alzheimer's Association, 2016). Mounting evidence implicates soluble aggregates of the $A \beta$ peptide, along with hyperphosphorylated Tau, as the main neurotoxins in $\mathrm{AD}$

\footnotetext{
* Corresponding author.

E-mail address: sebollela@fmrp.usp.br (A. Sebollela).
} 
(reviewed in Glabe, 2006; Ferreira and Klein, 2011; Viola and Klein, 2015). However, the complexity of the molecular and cellular mechanisms underlying $\mathrm{AD}$ has hampered the development of effective diagnostic and therapeutic approaches (Mucke and Selkoe, 2012; Nelson and Alafuzoff, 2012; Puzzo and Gulisano, 2015a, 2015b). Conceivably, this limited progress is in part due to the extensive use of disease models such as transgenic mice overexpressing the amyloid precursor protein, APP, which do not fully recapitulate the complexity of sporadic AD mechanisms (Puzzo and Gulisano, 2015a, 2015b). In this context, the availability of slice cultures from adult human brain is of great interest.

Organotypic cultures, herein termed slice cultures, have been defined as three-dimensional tissue cultivated on, or within, a gel support (e.g., collagen or artificial ECM) or free floating in culture medium (Hoffman, 2001). Slice cultures from different tissues have been reported, including skin, lung and brain (Hoffman, 2001; Steinstraesser and Rittig, 2009; Humpel, 2015). The main advantage of slice cultures over dissociated cell cultures is the preservation of the original tissue architecture and connections. This feature has key implications for the application of tissue slice cultures in bioscience. Notably, slice cultures are more resistant to the action of some drugs or toxins than dissociated, 2D-cultures (Miller and Miller, 1985; Elkayam and AmitayShaprut, 2006; Olinga and Schuppan, 2013), and respond differently to physiological stimuli such as insulin compared to organotypic cultures from liver tissue (Dash and Figler, 2017).

Classic protocols for culturing post-natal rodent brain slices were described many years ago (Gahwiler, 1981; Stoppini and Buchs, 1991), and have been the basis for the development of innovative protocols. More recently, successful cultures of adult human brain tissue using either postmortem or surgical explants have been reported (Brewer and Espinosa, 2001; Verwer and Hermens, 2002; Dai and Buijs, 2004; Freiman and Surges, 2006; Risher and Lee, 2011; Sebollela and FreitasCorrea, 2012; Eugene and Cluzeaud, 2014). Notably, amygdala-hippocampectomy has been the source of tissue in most of the papers using slice cultures from non-postmortem adult human brain (e.g. Brewer and Espinosa, 2001; Sebollela and Freitas-Correa, 2012; Eugene and Cluzeaud, 2014; Schwarz and Hedrich, 2017), which is mostly due to the necessary removal of a non-epileptogenic area (temporal cortex) to provide access to the mesial structures to be resected. In those studies, tissue remained alive in culture for days to weeks, and two studies showed preserved electrical activity (Eugene and Cluzeaud, 2014). Despite some efforts aimed at characterizing the health and functionality of those slice cultures (reviewed in Jones and da Silva, 2016), limited information is currently available on adult human brain-derived histocultures. This is surprising considering that cultured adult human brain tissue should represent a preferred model for studying age-associated neurodegenerative diseases (Przedborski and Vila, 2003), as they are potentially free from artifacts introduced when postmortem tissue is used (Born and Matos, 2017) or derived from loss of the original neuronal connectivity in dissociated cultures.

Here our goal was to characterize the cell content, viability and neural activity in adult human brain-derived slices cultivated freefloating for short-term. This method distinguishes from previously reported protocols focused on long-term culturing, which usually require the use of customized media and semiporous membranes. In addition, we evaluated the potential of such cultures to serve as a platform to study molecular events in age-associated human neuropathologies, using as a model the impact of $\mathrm{AD}$-associated $\mathrm{A} \beta$ oligomers (Viola and Klein, 2015). We found that the majority of neurons in cultured slices are alive and neurochemically active up to at least four days in vitro, which allowed the detection of binding of $A \beta$ oligomers. This study may help to broaden the application of slice cultures derived from adult human brain in studies of mechanisms of pathogenesis, with the potential to enhance the translational impact of novel therapeutics for human brain disorders.

\section{Methods}

\subsection{Human samples}

Cortical tissue was obtained from adult patients submitted to amygdalo-hippocampectomy for the treatment of refractory temporal lobe epilepsy (TLE), in collaboration with the Epilepsy Surgery Center (CirEp) at the Clinical Hospital of the Ribeirão Preto Medical School University of São Paulo, or at the University Hospital at the Federal University of Rio de Janeiro. Preoperative mapping of epileptogenic foci was carried out by magnetic resonance imaging and video coupled to electroencephalography (video-EEG). In most cases, the epileptogenic focus was restricted to the mesial zone of the temporal lobe. In one patient, epileptogenic activity (EEG) was also observed at the lateral (neocortical) zone of the temporal lobe. Tissue was obtained from 33 adults ( $55 \%$ male) averaging $40.5 \pm 11.3$ years old, and comprised a $1 \mathrm{~cm}^{3}$ sampling of the lateral temporal cortex which is removed to access the mesial structures of the temporal lobe. This study was approved both by the Ribeirão Preto Medical School and the Federal University of Rio de Janeiro Ethics Committees (HCRP \#17578/15 and UFRJ \#0069.0.197.000-05).

\subsection{Tissue culture}

The following procedure is a modification of the protocol described in Sebollela and Freitas-Correa (2012). Briefly, a fragment of lateral temporal cortex was collected at the surgical room immediately after resection, immersed in ice-cold oxygenated medium [50\% v/v HBSS (Hank's Balanced Salt Solution) in Neurobasal A (Gibco) supplemented with $10 \mathrm{mM}$ Hepes and glucose $3 \mathrm{mg} / \mathrm{ml}$ ] and transported to the Laboratory in approximately $15 \mathrm{~min}$. Tissue was immersed in ice-cold oxygenated HBSS, pia mater was carefully removed, and the remaining fragment was sliced at $200 \mu \mathrm{m}$ in a VT1000 s automatic vibratome (Leica) in such a way that each slice contained all the cortical layers and white matter. Slices were transferred to a Petri dish with cold oxygenated HBSS for trimming in an approximate ratio of $1 / 3$ white matter to $2 / 3$ grey matter, and plated in 24-well plates ( 1 slice/well) filled with $600 \mu \mathrm{L}$ of Neurobasal A (Gibco) supplemented with 1\% Glutamax (Gibco), 1\% Penicillin/Streptomycin (Gibco), 2\% B27 (Gibco) and $025 \mu \mathrm{g} / \mathrm{mL}$ Amphotericin B (Gibco). Free-floating cultures were incubated at $36{ }^{\circ} \mathrm{C}$ and $5 \% \mathrm{CO}_{2}$. By the end of day $0,200 \mu \mathrm{L}$ of the medium were replaced by fresh medium supplemented with $50 \mathrm{ng} / \mathrm{mL}$ BDNF (Sigma Aldrich). At day in vitro (DIV) 1, the volume of medium per well was reduced to $400 \mu \mathrm{L}$ and thereafter one third of the volume was replaced by BDNF-supplemented fresh medium every $24 \mathrm{~h}$.

\subsection{Cell viability in cultured slices}

Viability was assessed using the MTT [3-(4,5-dimethylthiazol-2 yl) -2,5-diphenyl tetrazoline bromide] assay (Mosmann, 1983). Briefly, MTT $(50 \mu \mathrm{g} / \mathrm{mL})$ was added at the indicated times and allowed to be metabolized for $3 \mathrm{~h}$ at $36^{\circ} \mathrm{C}$. Next, the slices were washed with PBS and frozen. Just before spectrophotometric reading, slices were homogenized in $200 \mu \mathrm{l}$ isopropanol/HCl $0.01 \mathrm{~N}$ using a motorized pestle (Kimble Chase) and centrifuged at $1000 \mathrm{rpm}$ for $1 \mathrm{~min}$. Supernatants were collected and the optical density (O.D.) at $540 \mathrm{~nm}$ was determined using a microplate reader (Molecular Devices). O.D. values were normalized by the mass of each slice.

\subsection{Histological analysis}

Slices were fixed in buffered paraformaldehyde (4\%) overnight at $4{ }^{\circ} \mathrm{C}$, cryoprotected with $30 \%$ sucrose, frozen and cut at $30 \mu \mathrm{m}$ in a freezing microtome (Leica). Sections were transferred to 24-well plates for free-floating Nissl staining with thionin as previously described (Kadar and Wittmann, 2009) or immunostaining. Immunostainings 
were performed as previously described (Horta-Junior Jde and Lopez, 2008) with modifications. Briefly, sections were incubated for $40 \mathrm{~min}$ in $2 \%$ normal donkey serum in phosphate buffer and incubated overnight with primary antibodies, anti-NeuN (Millipore, MAB377; 1:1000 dilution), anti-GFAP (Novocastra, NCL-GFAP-GA5; 1:1000 dilution) or antiIba1 (Abcam, ab178846; 1:2000 dilution). After incubation with avidin-biotin secondary antibodies (Vectastain, Kit Standard, PK-4000, Vector), immunoreactivity was revealed using $\mathrm{DAB}+0.04 \%$ nickel ammonium. Finally, sections were mounted on gelatin coated slides, air dried, dehydrated in increasing ethanol solutions, delipified in xylene, and imaged.

\subsection{Western blotting}

Extracts from 1 to 2 slices were prepared in RIPA buffer $(50 \mathrm{mM}$ Tris - HCL, pH 7.4; $150 \mathrm{mM} \mathrm{NaCl;} 1.5 \mathrm{mM} \mathrm{MgCl}$; $1.5 \mathrm{mM}$ EDTA; $1 \%$ Triton X-100; $10 \%$ glycerol; supplemented with protease and phosphatase inhibitors) using a motorized pestle. Homogenates were centrifuged at $4{ }^{\circ} \mathrm{C}$ for $10 \mathrm{~min}$ at $16,000 \mathrm{x} \mathrm{g}$ and supernatants were collected. Protein content was determined using the Bradford reagent (BioRad) and $50 \mu \mathrm{g}$ were resolved on a $12 \%$ SDS-PAGE. The proteins were transferred to a nitrocellulose membrane using the transblot TURBO apparatus (Bio-Rad). The membrane was blocked at RT for $1 \mathrm{~h}$ with $5 \%$ non-fat dry milk in TBS $(0.05 \mathrm{M}$ Tris, $0.15 \mathrm{M} \mathrm{NaCl}, \mathrm{pH} 7.5)$ plus $0.1 \%$ Tween 20 (Sigma-Aldrich), and incubated overnight with antiNeuN (1:1000; MAB377, Millipore), and $\beta$-actin (1:10,000; MAB1501, Millipore). Primary antibodies were diluted in TTBS with $2.5 \%$ BSA (Sigma-Aldrich). After washing and incubation with HRP-conjugated secondary antibodies (GE Healthcare), membranes were developed using ECL-Prime (GE Healthcare). When necessary, membranes were stripped with 1\% SDS, $100 \mathrm{mM}$ glycine, 0,1\% NP40, pH 2.0 for $40 \mathrm{~min}$ at RT and re-blocked before incubation with primary antibody. Quantitative analyses were made using NHI ImageJ software.

\subsection{Neurotransmitter release assay}

Neuronal depolarization was induced by transferring the slices from HBSS ( $15 \mathrm{~min}$ at $37^{\circ} \mathrm{C}$, corresponding to basal levels of neurotransmitter) to HBSS plus $80 \mathrm{mM}$ of $\mathrm{KCl}\left(15 \mathrm{~min}\right.$ at $37^{\circ} \mathrm{C}$, corresponding to $\mathrm{KCl}$-induced released neurotransmitters). When indicated, slices were incubated with $\mathrm{Ca}^{2+}$-free HBSS. Media were collected and used for analysis of neurotransmitter levels. Both basal and KCl-evoked released GABA and L-glutamate were measured in slice culture media by high-performance liquid chromatography coupled to electrochemical detection (HPLC-ED, L-ECD-6 A module; Shimadzu), using a reversephase C-18 column (Kromasil; $5 \mu \mathrm{m}$ particle size, $250 \mathrm{~mm} \times 4.6 \mathrm{~mm}$ ). OPA-sulfite derivatives of amino acids were generated immediately before sample injection $(50 \mu \mathrm{L})$, as previously described (Monge-Acuna and Fornaguera-Trias, 2009). Standard curves were generated using Lglutamate and GABA standards (Sigma-Aldrich).

\subsection{Binding of $A \beta$ oligomers to human brain cultures}

$A \beta$ oligomers (A $\beta O s$ ) were prepared using $A \beta_{1-42}$ (American Peptide) as in Chromy and Nowak (2003). At DIV 2, cortical slices were treated with $\mathrm{A} \beta \mathrm{Os} 250 \mathrm{nM}$ or vehicle for $1 \mathrm{~h}$ at $37^{\circ} \mathrm{C}$, washed twice with medium, and immediately after either fixed with $4 \%$ paraformaldehyde for IHC or homogenized under non-denaturing conditions for ELISA. In both cases, samples were probed using the anti-A $\beta O$ scFv-type antibody, NUsc1 (Velasco, Heffern et al. 2012). For IHC, fixed tissue was paraffin-embedded and cut at $5 \mu \mathrm{m}$. Thin sections were incubated with phage-bound NUsc1 (pbNUsc1) followed by HRP-conjugated antiphage M13 (GE). Reactivity was revealed using DAB. ELISA was performed as in Sebollela et al. (2017). Briefly, 96-well plates were coated overnight with $100 \mu \mathrm{L}$ monoclonal anti-pan $\mathrm{A} \beta$ antibody (6E10, Covance) at $1.0 \mu \mathrm{g} / \mathrm{mL}$ in PBS at $4{ }^{\circ} \mathrm{C}$, washed and blocked with $200 \mu \mathrm{L}$ of
2\% NFD milk/PBS. Detergent-free brain slice extracts were added at $0.5 \mathrm{mg} / \mathrm{mL}$ (total protein) diluted in $2 \%$ NFD milk/PBS and incubated overnight at $4{ }^{\circ} \mathrm{C}$, followed by washing with PBS containing $0.05 \%$ Tween-20, and incubated with NUsc1 (diluted 1:100 in 2\% milk-PBS) for $2 \mathrm{~h}$ at RT. Detection of bound antibody was achieved by incubation with HRP-conjugated anti-phage M13 (GE). Plates were developed using TMB (Sigma) as substrate. OD was read at $450 \mathrm{~nm}$ in a plate reader (Molecular Devices).

\subsection{Total tau and p-tau levels in human brain cultured slices exposed to A $\beta$ oligomers}

Human brain slices at DIV7 were incubated with A 30 s $(500 \mathrm{nM})$ for $24 \mathrm{~h}$, washed and homogenized in RIPA buffer containing protease inhibitors cocktail (Sigma-Aldrich) and $100 \mathrm{mM}$ of the phosphatase inhibitor, sodium orthovanadate (Sigma-Aldrich), with a motorized pestle (Kimble Chase). A $12 \%$ SDS-PAGE was loaded with $20 \mu \mathrm{g}$ of total protein. Western blotting was carried out as described above, and membranes were incubated with anti-p-Tau Ser396 rabbit polyclonal (1:1000; Santa Cruz Biotechnology), p-Tau Ser202/Thr205 mouse monoclonal (1:1000; Thermo Fisher Scientific), Tau-1 mouse monoclonal (1:1000; Merck-Millipore) and $\beta$-Actin mouse monoclonal (1:10,000; Santa Cruz Biotechnology). Quantitative analysis was made using ImageJ software (Schneider et al., 2012). The basal level (100\%) of Tau phosphorylation was determined in slices treated with vehicle.

\subsection{Statistical analysis}

Statistical analysis was performed using Graph Pad Prism 6 (Graph Pad Software). Means were compared using either Student's $t$-test or one-way ANOVA, as indicated in the legends for the figures. The number of donors has been established as the experimental unit (referred to as "N" throughout the text).

\section{Results and discussion}

\subsection{Cell viability and tissue structure in cultured adult human brain slices}

We initially employed the MTT assay, which has been successfully used to assess cell viability in a variety of histocultures (Tobita and Izumi, 2010; Wang and Peng, 2010; Paterniti and Impellizzeri, 2013), to evaluate cell viability in adult human brain slices along four days in culture. MTT reduction was not altered up to 4 days in vitro (DIV), indicating no extensive reduction in cell viability (Fig. 1A). A trend of decrease (albeit not statistically significant) in MTT reduction was verified at $4 \mathrm{DIV}$, suggesting that extending the culture period longer could result in reduced cell viability. Indeed, preliminary MTT data at DIV9 ( $\mathrm{N}=2$ donors) indicate that viability at this stage is approximately 50\% compared to DIV0 (data not shown). Preservation of cell viability up to 4 DIV obtained with the current protocol is likely a consequence of BDNF supplementation (Supp. Fig. 1) and the reduction in the thickness of the slices to $200 \mu \mathrm{m}$, compared to often used 300-400 $\mu \mathrm{m}$ slices (Sebollela and Freitas-Correa, 2012; Eugene and Cluzeaud, 2014), which may have a positive impact on diffusion of $\mathrm{O}_{2}$ and nutrients through the tissue. We have also observed that the rate of medium consumption (determined by acidity) dropped markedly by the end of DIV1, likely as a consequence of the metabolic recovery from processing and stabilization to the in vitro environment. Thus, we preferentially used DIV2 or later for treatments.

In line with preservation of cell viability, tissue architecture was not affected by tissue processing or by cultivation (Fig. 1B-D). Neuronal content in slices was assessed by both IHC and Western blotting (WB) for neuronal nuclear protein NeuN, a typical marker of mature and healthy neurons (Mullen and Buck, 1992). Neuronal morphology and tissue organization were preserved in cultured slices (Fig. 2A-D). In addition, WB analysis revealed no significant differences in NeuN levels 
A

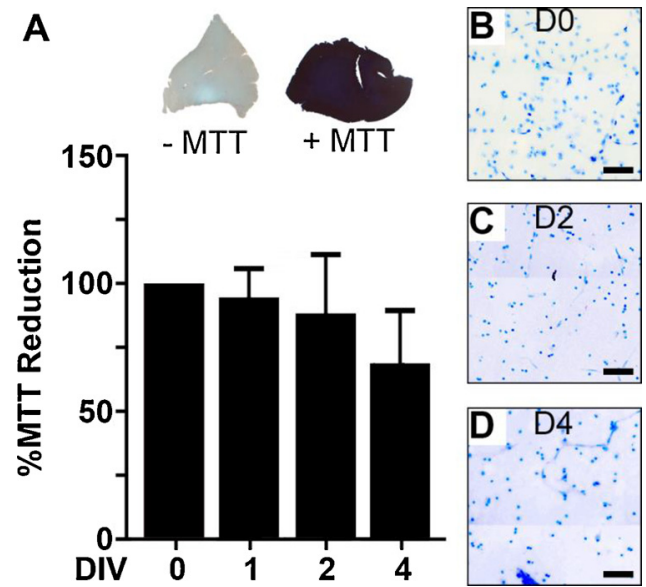

Fig. 1. Cell viability and tissue structure in slice cultures from adult human cortex. (A) Viability in slices kept in culture for the indicated times. DIV: days in vitro. Viability was evaluated by the MTT assay. Representative slices incubated (dark blue) or not (white) with MTT are shown. Absorbance values were normalized by the mass $(\mathrm{mg})$ of each slice. ( $\mathrm{N}=4-7$ individual tissue donors for each time point). Data are plotted as means \pm standard error. p-values for all comparisons were $>0.05$ (one-way ANOVA). (B-D) Cytoarchitecture was assessed by Nissl staining at days 0, 2 and 4. Images correspond to cortical layers II/III. Scale bar $=50 \mu \mathrm{m}$. (For interpretation of the references to colour in this figure legend, the reader is referred to the web version of this article).

along the period in culture (Fig. 2E-F). We note that the NeuN labeling pattern varied amongst donors, in some cases presenting bands migrating at MW higher than $66 \mathrm{kDa}$, believed to be non-specific (Darlington and Goldman, 2008) and, for this reason, not included in quantification. In combination, IHC and WB results indicate preservation of neuronal number and viability in cultured slices. Importantly, the presence of astrocytes and microglia, expected if tissue is indeed preserved, were both confirmed in slices at DIV4 (Fig. 2 G-H). Taken together, these data indicate that our free floating slices resemble the original in vivo environment, a key motivation for developing the current method. This is particularly relevant considering that slices cultured on membranes are known to undergo flattening and synaptic re-organization upon long-term cultivation in newborn rodent brain- derived cultures (Humpel, 2015; Gilbride, 2016), and changes in gene/ protein expression in the rodent mature brain (Staal and Alexander, 2011). Therefore, although flattening has not been reported in recent works using long-term adult human brain-derived slice cultures (Eugene and Cluzeaud, 2014; Andersson and Avaliani, 2016), morphofunctional alterations triggered by plasticity-like events associated to the adaptation to the in vitro environment should not be completely ruled out. Supporting this notion is the observation by Eugene and Cluzeaud (2014) of a slight difference in electrophysiological patterns between slices at 9DIV and 24DIV. Altogether, it is possible to suggest that short-term human brain slice cultures might model with more accuracy the actual response of the mature human brain to neurotoxic challenges, such as the accumulation of $\mathrm{A} \beta$ oligomers in $\mathrm{AD}$, than longterm organotypic cultures. Furthermore, by eliminating the use of membranes, we have significantly simplified and reduced the total cost of culturing this valuable tissue, a relevant aspect if one considers running high content drug screening studies.

\subsection{Functional analysis in cultured human brain tissue}

In order to assess neurotransmitter release in the cultures, slices were subjected to $\mathrm{KCl}$-induced depolarization followed by determination of neurotransmitter release to the medium. As expected, at 0 DIV, depolarization by $\mathrm{KCl}$ induced a greater than 5 -fold increase in the release of the major excitatory and inhibitory neurotransmitters, glutamate and GABA, respectively (Fig. 3). Importantly, KCl-evoked release of neurotransmitters was preserved up to 4 DIV (Fig. 3).

To analyze the contribution of vesicular-dependent release to the global neurotransmitter release measured under our conditions, we carried out $\mathrm{KCl}$ depolarization in $\mathrm{Ca}^{2+}$-free medium. Results indicate that neurotransmitter release using this protocol was mainly synaptic, since release in the absence of calcium was significantly reduced at both 1 and 2 DIV (Fig. 3, white bars). Collectively, these data suggest that neuronal activity in cultured slices is preserved at least until 4 DIV. In addition, considering the known role of astrocytes in the modulation of synaptic neurotransmitter release (Wallach and Lallouette, 2014; Sobieski and Jiang, 2015; Cazemier and Clascá, 2016), and of microglial cells in the formation and maintenance of neuronal synapses (Harry, 2013; Lim and Park, 2013), these functional data suggest that the main cell connections in the cortex are preserved in adult human
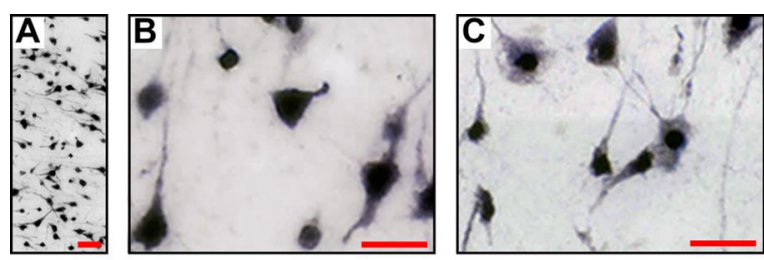

E

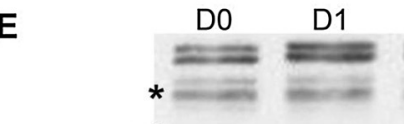

\begin{abstract}
$\mathrm{D} 2$
\end{abstract}
D4
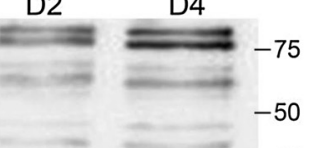

$-37$

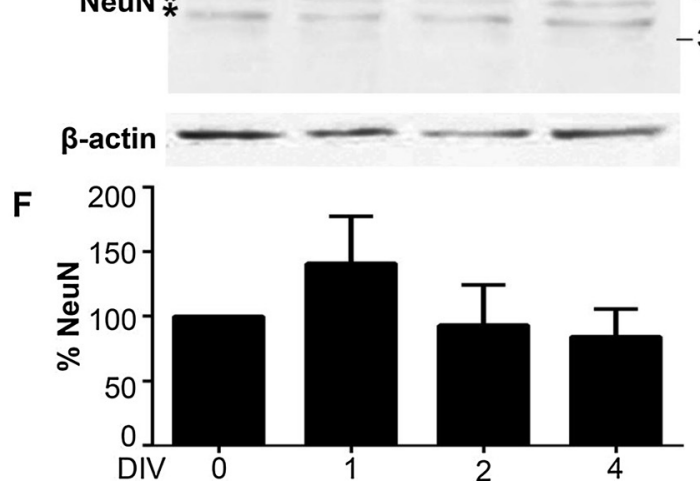

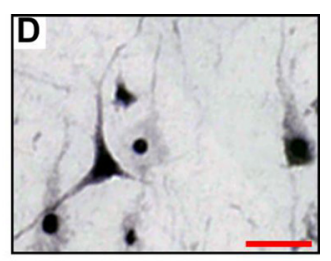
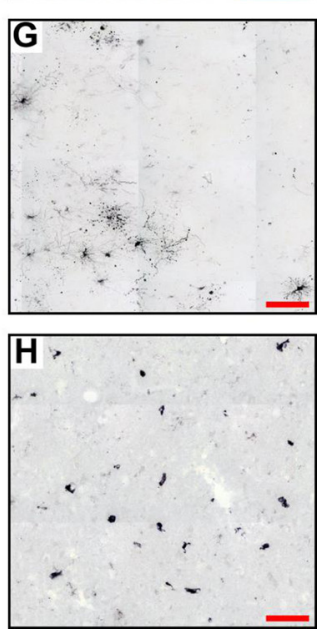

Fig. 2. Neural cell content in adult human brain slices in culture. Neurons were identified by NeuN immunohistochemistry in cultured slices at day 0 (A and B), 1 (C) and 4 (D). Neuronal content along period in culture was quantified by Western Blotting for NeuN. A representative membrane is shown in $\mathrm{E}$, and quantification (normalized by $\beta$ actin levels) is shown in $\mathrm{F}(\mathrm{N}=3-7$ independent human tissue donors for each time point). p-values for all comparisons were $>0.05$ (one-way ANOVA). Astrocytes and microglia were also identified by GFAP (G) or Ibal (H) immunostaining, respectively, at day 4. All images correspond to cortical layer V. Scale bars correspond to $100 \mu \mathrm{m}$ (A, G, H) or $25 \mu \mathrm{m}$ (B, C, D). 


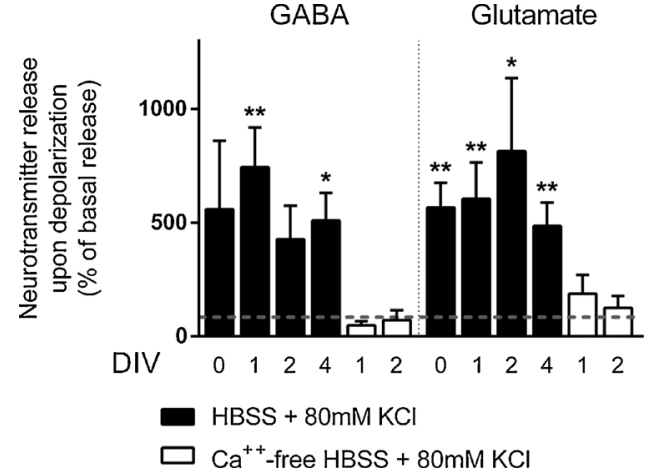

Fig. 3. Neurotransmitter release in adult human brain slices in culture. GABA and glutamate release were determined after $\mathrm{KCl}$ depolarization. Slices incubated in the presence of $\mathrm{KCl}$ (black bars) presented higher levels of both neurotransmitters compared to the basal release (indicated by the horizontal dashed line) at all time points tested. When $\mathrm{KCl}$ depolarization was performed in $\mathrm{Ca}^{++}$-free medium (white bars), the $\mathrm{KCl}$-evoked neurotransmitter release was markedly reduced at both DIV 1 and 2 . $\mathrm{N}=3-8$ individual tissue donors for each time point. *p $<0.05$; $* * \mathrm{p}<0.01$ (one-way ANOVA).

brain slices in culture. Although we have not directly investigated the maintenance and extension of functional brain circuits in cultured slices, in a previous study, Eugene and Cluzeaud (2014) have reported the occurrence of spontaneous action potentials in adult human cortical slices after 9 DIV.

A major concern with the use of cortical tissue ressected from TLE cases to prepare slice cultures is the possibility that neurophysiological alterations might have spreaded from the hippocampal focus of epileptic activity to the neocortical region (lateral) which is the source of the cultivated tissue. The concept of epilepsy as a network disease, or the concept of focal epilepsy, with potential secondary spreading (kindling effect; mirror foci or secondary epileptogenesis) are of paramount importance to the potential use of the surgical tissue in translational neuroscience (Trevelyan, 2016).

Although no clear data on this is yet available, some authors have provided clues on this issue. Using acute slices from both epileptic and non-epileptic donors, Freiman and Surges (2006) observed alterations on $\mathrm{K}_{\mathrm{ATP}}$ channel function but not in the response of calcium channels. The work from Verhoog and Goriounova (2013) showed no significant difference in electrical properties between acute slices from similar brain regions derived from human temporal lobe epilepsy (TLE), brain tumor or malformation patients or rats. More recently, Eugene and Cluzeaud (2014) used acute cortical slices from adult human brain obtained from TLE cases and detected no spontaneous interictal-like activity. Combined with our current results showing the expected response in terms of $\mathrm{K}^{+}$-induced release of glutamate and GABA, those previous observations suggest that cortical slices (distal to the epileptic foci) prepared from TLE patients are neurochemically normal and thus hold potential to be used as a preferred model for studying age-associated brain diseases. It is important to note, however, that the feasibility of using short-term cultures in free floating format in electrophysiological studies needs to be further investigated, and compared with the encouraging results obtained using customized media and semiporous membranes reported by Eugene and Cluzeaud (2014) and Schwarz and Hedrich (2017).

\subsection{Modeling Alzheimer's disease using cultured slices from adult human brain}

Since we were able to culture adult human brain slices from different donors, and to keep them alive and synaptically active for at least 4 DIV, we asked whether this histoculture could be used as a platform to model sporadic $\mathrm{AD}$ in bona fide human brain tissue. A key motivation for this analysis comes from the work from Verhoog and Goriounova (2013) showing that acute (used within $15 \mathrm{~h}$ post-surgery) neocortical slices derived from epileptic patients presented no sign of spontaneous epileptiform activity, suggesting that slice cultures from this brain region would be useful in the study of several neurological diseases. Since it is generally accepted that sporadic $\mathrm{AD}$ is linked to brain accumulation of $\mathrm{A} \beta$ oligomers (A $\mathrm{\beta O}$ ), which in turn leads to Tau hyperphosphorylation (De Felice and Wu, 2008; Forny-Germano and Lyra e Silva, 2014; Selkoe and Hardy, 2016), we exposed cultures to exogenous $\mathrm{A} \beta \mathrm{O}$, an approach that have been widely applied to either neural cultures or animals (by icv injection) to model AD pathology (reviewed in Benilova and Karran, 2012; Walsh and Teplow, 2012; Viola and Klein, 2015). A BO binding to the tissue was measured by ELISA in extracts prepared from slices incubated with either vehicle or $A \beta$ oligomers. Robust binding of $\mathrm{A} \beta \mathrm{O}$ s to cultured brain slices was evident (Fig. 4A). Binding was specific for $A \beta$ oligomers, as we have used as detecting antibody the conformational scFv-type antibody NUsc1, shown to be highly specific towards AßOs (Velasco and Heffern, 2012; Sebollela and Cline, 2017). A 30 binding to adult human brain slices was further detected by IHC using tissue from a different donor. Intense NUsc1-reactive labeling associated to cells was observed only in tissue exposed to $\mathrm{A} \beta$ oligomers (Fig. $4 \mathrm{~B}-\mathrm{C}$ ). Interestingly, this labeling pattern resembles that observed in $\mathrm{AD}$ human brain sections probed with antiA $\beta$ oligomer antibodies (Lacor and Buniel, 2004).

Modeling the neurotoxicity of $\mathrm{AD}$-associated $\mathrm{A} \beta$ oligomers using adult human brain slice cultures has been previously attempted. Alterations in gene expression (Sebollela and Freitas-Correa, 2012) and
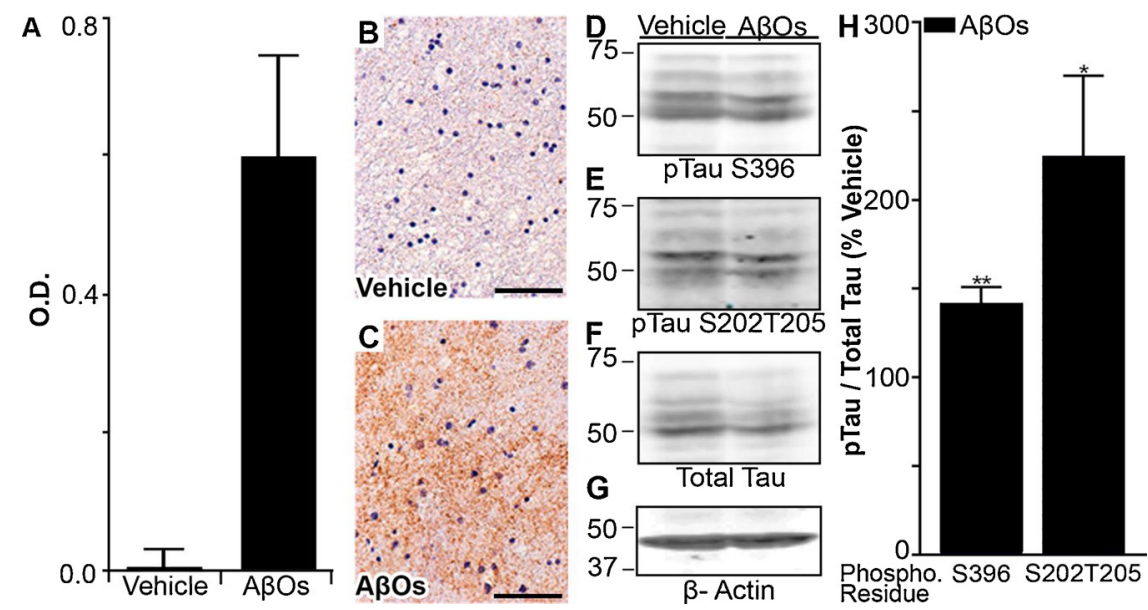

Fig. 4. Toxicity of Alzheimer's-associated A $\beta$ oligomers to human brain slices in culture. Slices were exposed to either vehicle or $A \beta$ oligomers $(250 \mathrm{nM}, 1 \mathrm{~h}$ ) and binding was evaluated using the anti-A $\beta$ oligomer scFv antibody, NUsc1, by both ELISA and IHC. Robust A 30 binding was readily detected in tissue homogenates by ELISA (A) and was also visualized by IHC in slices prepared from a different donor (B, C). Signal was developed using DAB. Nuclei are stained with hematoxylin. Scale bar $=50 \mu \mathrm{m}$. Phosphorylated Tau (pTau) levels were determined in vehicle or A $\mathrm{BO}$-treated cultured slices using tissue from an independent set of donors $(\mathrm{N}=5)$. Slices were treated with vehicle or $\mathrm{A} \beta \mathrm{O}$ s $(500 \mathrm{nM})$ for $24 \mathrm{~h}$, homogenized and assayed by Western Blotting probing for two different pTau epitopes (S396 and S202T205), total Tau, and $\beta$ actin as loading control. Representative blots are shown in D-G. pTau levels (bands between 50 and $75 \mathrm{KDa}$ ) were normalized to total Tau and plotted as percentage of pTau in vehicle-treated slices $(\mathrm{H})$. Two asterisks denote

$\mathrm{p}<0.01$ and one asterisk denote $\mathrm{p}<0.05$ (Student's t-test). 
energy metabolism (Seixas da Silva and Melo, 2017) induced by AßOs in adult human brain slice cultures have been reported. In AD brains, it is though that brain accumulation of $\mathrm{A} \beta \mathrm{O}$ s leads to hyperphosphorylation of the microtubule associated protein, Tau, which is a hallmark of AD (Selkoe, 2001; Kowalska, 2004; Iqbal and Alonso Adel, 2005). This prompted us to investigate whether exposure of cultured human brain slices to A $\beta O$ s would trigger Tau hyperphosphorylation. Results showed a decreased in total Tau levels compared to $\beta$-actin in slices exposed to A 30 s (Fig. 4F-G). Interestingly, pTau/Tau ratio was higher in $\mathrm{A} \beta \mathrm{O}$-treated slices, ranging from 142 to $225 \%$ depending on the phosphoepitope examined (Fig. $4 \mathrm{H}$ ). It is important to point out that this increase was observed at phosphorylation sites strongly associated with AD (Iqbal and Alonso Adel, 2005). These results suggest that adult human brain slice cultures prepared as described herein are amenable to model molecular/cellular alterations in AD. Further, such cultures represent an advance over previously described cultures prepared from both fetal or postmortem human brains, in which either cell connections or the molecular machinery are quite different from that in adult live brain parenchyma, considering that fetal tissue presents cellular connections not yet fully developed (Mountcastle, 1997; Rakic, 2009; Lui and Hansen, 2011), while the later undergoes biochemical and neuroanatomical changes from death to tissue collection (Fountoulakis and Hardmeier, 2001; Lavenex and Lavenex, 2009). Studies on the feasibility of using these slice cultures to model other age-associated neurodegenerative diseases, such as Parkinson Disease, should now be pursued.

\section{Conclusions}

In the present work, we describe a detailed protocol for culturing adult human brain slices obtained from surgical explants in freefloating format, which renders viable and neurochemically functional slices for at least four days in culture. We have found that these slices are sensitive to Alzheimer's disease-associated A $\beta$ oligomers, suggesting that this adult CNS model may be a useful tool to study molecular and cellular mechanisms of neurodegenerative diseases in a more clinically relevant context.

\section{Acknowledgments}

This work was supported by grants from Fundação de Amparo a Pesquisa do Estado de Sao Paulo (FAPESP) and FAEPA to AS and NGC; National Institute for Translational Neuroscience, Fundação de Amparo a Pesquisa do Estado do Rio de Janeiro (FAPERJ) and Conselho Nacional de Desenvolvimento Cientifico e Tecnológico (CNPq) to STF and FGDF; and Coordenação de Aperfeiçoamento de Pessoal de Nível Superior (CAPES). We thank Deise L Chesca and Silvana El-Chaedroui, and CIREP staff (especially Tânia A. C. Becker and Adriana A. C. Ferrari) for excellent technical assistance. NGC holds a CNPq Researcher fellowship.

\section{Appendix A. Supplementary data}

Supplementary material related to this article can be found, in the online version, at doi:https://doi.org/10.1016/j.jneumeth.2018.05. 021.

\section{References}

Alzheimer's Association, 2016. Alzheimer's disease facts and figures. Alzheimers Dement 12, 459-509.

Andersson, M., Avaliani, N., et al., 2016. Optogenetic control of human neurons in organotypic brain cultures. Sci. Rep. 6, 24818.

Benilova, I., Karran, E., et al., 2012. The toxic Abeta oligomer and Alzheimer's disease: an emperor in need of clothes. Nat. Neurosci. 15 (3), 349-357.

Born, J.P.L., Matos, H.C., et al., 2017. Using postmortem hippocampi tissue can interfere with differential gene expression analysis of the epileptogenic process. PLoS One
12 (8).

Brewer, G.J., Espinosa, J., et al., 2001. Culture and regeneration of human neurons after brain surgery. J. Neurosci. Methods 107 (1-2), 15-23.

Cazemier, J.L., Clascá, F., et al., 2016. Connectomic analysis of brain networks: novel techniques and future directions. Front. Neuroanat. 10, 110.

Chromy, B.A., Nowak, R.J., et al., 2003. Self-assembly of Abeta(1-42) into globular neurotoxins. Biochemistry 42 (44), 12749-12760.

Dai, J., Buijs, R., et al., 2004. Glucocorticoid hormone (cortisol) affects axonal transport in human cortex neurons but shows resistance in alzheimer's disease. Br. J. Pharmacol. 143 (5), 606-610.

Darlington, P.J., Goldman, J.S., et al., 2008. Widespread immunoreactivity for neuronal nuclei in cultured human and rodent astrocytes. J. Neurochem. 104 (5), 1201-1209.

Dash, A., Figler, R.A., et al., 2017. Pharmacotoxicology of clinically-relevant concentrations of obeticholic acid in an organotypic human hepatocyte system. Toxicol. In Vitro 39, 93-103.

De Felice, F.G., Wu, D., et al., 2008. Alzheimer's disease-type neuronal tau hyperphosphorylation induced by A beta oligomers. Neurobiol. Aging 29 (9), 1334-1347.

Elkayam, T., Amitay-Shaprut, S., et al., 2006. Enhancing the drug metabolism activities of C3A-a human hepatocyte cell line-by tissue engineering within alginate scaffolds. Tissue Eng. 12 (5), 1357-1368.

Eugene, E., Cluzeaud, F., et al., 2014. An organotypic brain slice preparation from adult patients with temporal lobe epilepsy. J. Neurosci. Methods 235, 234-244.

Ferreira, S.T., Klein, W.L., 2011. The Abeta oligomer hypothesis for synapse failure and memory loss in Alzheimer's disease. Neurobiol. Learn. Mem. 96 (4), 529-543.

Forny-Germano, L., Lyra e Silva, N.M., et al., 2014. Alzheimer's disease-like pathology induced by amyloid-beta oligomers in nonhuman primates. J. Neurosci. 34 (41), 13629-13643.

Fountoulakis, M., Hardmeier, R., et al., 2001. Postmortem changes in the level of brain proteins. Exp. Neurol. 167 (1), 86-94.

Freiman, T.M., Surges, R., et al., 2006. K(+)-evoked [(3)H]-norepinephrine release in human brain slices from epileptic and non-epileptic patients is differentially modulated by gabapentin and pinacidil. Neurosci. Res. 55 (2), 204-210.

Gahwiler, B.H., 1981. Organotypic monolayer cultures of nervous tissue. J. Neurosci. Methods 4 (4), 329-342.

Gilbride, C.J., 2016. The hyperexcitability of dentate granule neurons in organotypic hippocampal slice cultures is due to reorganization of synaptic inputs in vitro. Physiol. Rep. 4 (19), 12889.

Glabe, C.G., 2006. Common mechanisms of amyloid oligomer pathogenesis in degenerative disease. Neurobiol. Aging 27 (4), 570-575.

Harry, G.J., 2013. Microglia during development and aging. Pharmacol. Ther. 139 (3), 313-326.

Hoffman, R.M., 2001. Histocultures and Organ Cultures. eLS. John Wiley \& Sons, Ltd.

Horta-Junior Jde, A., Lopez, D.E., et al., 2008. Direct and indirect connections between cochlear root neurons and facial motor neurons: pathways underlying the acoustic pinna reflex in the albino rat. J. Comp. Neurol. 507 (5), 1763-1779.

Humpel, C., 2015. Organotypic brain slice cultures: a review. Neuroscience 305, 86-98.

Iqbal, K., Alonso Adel, C., et al., 2005. Tau pathology in Alzheimer disease and other tauopathies. Biochim. Biophys. Acta 1739 (2-3), 198-210.

Jones, R.S., da Silva, A.B., et al., 2016. Human brain slices for epilepsy research: pitfalls, solutions and future challenges. J. Neurosci. Methods 260, 221-232.

Kadar, A., Wittmann, G., et al., 2009. Improved method for combination of immunocytochemistry and Nissl staining. J. Neurosci. Methods 184 (1), 115-118.

Kowalska, A., 2004. [The beta-amyloid cascade hypothesis: a sequence of events leading to neurodegeneration in Alzheimer's disease]. Neurol Neurochir. Pol. 38 (5), 405-411.

Lacor, P.N., Buniel, M.C., et al., 2004. Synaptic targeting by Alzheimer's-related amyloid beta oligomers. J. Neurosci. 24 (45), 10191-10200.

Lavenex, P., Lavenex, P.B., et al., 2009. Postmortem changes in the neuroanatomical characteristics of the primate brain: hippocampal formation. J. Comp. Neurol. 512 (1), 27-51.

Lim, S.-H., Park, E., et al., 2013. Neuronal synapse formation induced by microglia and interleukin 10. PLoS One 8 (11), e81218.

Lui, J.H., Hansen, D.V., et al., 2011. Development and evolution of the human neocortex. Cell 146 (1), 18-36.

Miller, B.E., Miller, F.R., et al., 1985. Factors affecting growth and drug sensitivity of mouse mammary tumor lines in collagen gel cultures. Cancer Res. 45 (9), 4200-4205.

Monge-Acuna, A.A., Fornaguera-Trias, J., 2009. A high performance liquid chromatography method with electrochemical detection of gamma-aminobutyric acid, glutamate and glutamine in rat brain homogenates. J. Neurosci. Methods 183 (2), $176-181$.

Mosmann, T., 1983. Rapid colorimetric assay for cellular growth and survival: application to proliferation and cytotoxicity assays. J. Immunol. Methods 65 (1-2), 55-63.

Mountcastle, V.B., 1997. The columnar organization of the neocortex. Brain 120 (Pt 4), $701-722$.

Mucke, L., Selkoe, D.J., 2012. Neurotoxicity of amyloid beta-protein: synaptic and network dysfunction. Cold Spring Harb. Perspect Med. 2 (7), a006338.

Mullen, R.J., Buck, C.R., et al., 1992. NeuN, a neuronal specific nuclear protein in vertebrates. Development 116 (1), 201-211.

Nelson, P.T., Alafuzoff, I., et al., 2012. Correlation of alzheimer disease neuropathologic changes with cognitive Status: a review of the literature. J. Neuropathol. Exp. Neurol. 71 (5), 362-381.

Olinga, P., Schuppan, D., 2013. Precision-cut liver slices: a tool to model the liver ex vivo. J. Hepatol. 58 (6), 1252-1253.

Paterniti, I., Impellizzeri, D., et al., 2013. A new co-ultramicronized composite including palmitoylethanolamide and luteolin to prevent neuroinflammation in spinal cord injury. J. Neuroinflammation 10, 91. 
Przedborski, S., Vila, M., et al., 2003. Series introduction: neurodegeneration: what is it and where are we. J. Clin. Invest. 111 (1), 3-10.

Puzzo, D., Gulisano, W., et al., 2015a. The keystone of Alzheimer pathogenesis might be sought in Abeta physiology. Neuroscience 307, 26-36.

Puzzo, D., Gulisano, W., et al., 2015b. Rodent models for Alzheimer's disease drug discovery. Expert Opin. Drug. Discov. 10 (7), 703-711.

Rakic, P., 2009. Evolution of the neocortex: a perspective from developmental biology. Nat. Rev. Neurosci. 10 (10), 724-735.

Risher, W.C., Lee, M.R., et al, 2011. Dibucaine mitigates spreading depolarization in human neocortical slices and prevents acute dendritic injury in the ischemic rodent neocortex. PLoS One 6 (7), e22351.

Schwarz, N., Hedrich, U.B.S., et al., 2017. Human cerebrospinal fluid promotes long-term neuronal viability and network function in human neocortical organotypic brain slice cultures. Sci. Rep. 7 (1), 12249.

Sebollela, A., Cline, E.N., et al., 2017. A human scFv antibody that targets and neutralizes high molecular weight pathogenic amyloid-beta oligomers. J. Neurochem. 3 (10), 14118.

Sebollela, A., Freitas-Correa, L., et al., 2012. Amyloid-beta oligomers induce differential gene expression in adult human brain slices. J. Biol. Chem. 287 (10), 7436-7445.

Seixas da Silva, G.S., Melo, H.M., et al., 2017. Amyloid-beta oligomers transiently inhibit AMP-activated kinase and cause metabolic defects in hippocampal neurons. J. Biol. Chem. 292 (18), 7395-7406.

Selkoe, D.J., 2001. Alzheimer's disease: genes, proteins, and therapy. Physiol. Rev. 81 (2), 741-766.

Selkoe, D.J., Hardy, J., 2016. The amyloid hypothesis of Alzheimer's disease at 25 years. EMBO Mol. Med. 8 (6), 595-608.

Sobieski, C., Jiang, X., et al., 2015. Loss of Local Astrocyte Support Disrupts Action Potential Propagation and Glutamate Release Synchrony from Unmyelinated
Hippocampal Axon Terminals In Vitro. J. Neurosci. 35 (31), 11105-11117.

Staal, J.A., Alexander, S.R., et al., 2011. Characterization of cortical neuronal and glial alterations during culture of organotypic whole brain slices from neonatal and mature mice. PLoS One 6 (7), e22040.

Steinstraesser, L., Rittig, A., et al., 2009. A human full-skin culture system for interventional studies. Eplasty 9, e5.

Stoppini, L., Buchs, P.A., et al., 1991. A simple method for organotypic cultures of nervous tissue. J. Neurosci. Methods 37 (2), 173-182.

Tobita, T., Izumi, K., et al., 2010. Development of an in vitro model for radiation-induced effects on oral keratinocytes. Int. J. Oral Maxillofac. Surg. 39 (4), 364-370.

Trevelyan, A.J., 2016. Do Cortical circuits need protecting from themselves. Trends Neurosci. 39 (8), 502-511.

Velasco, P.T., Heffern, M.C., et al., 2012. Synapse-binding subpopulations of Abeta oligomers sensitive to peptide assembly blockers and scFv antibodies. ACS Chem. Neurosci. 3 (11), 972-981.

Verhoog, M.B., Goriounova, N.A., et al., 2013. Mechanisms underlying the rules for associative plasticity at adult human neocortical synapses. J. Neurosci. 33 (43), 17197-17208.

Verwer, R.W., Hermens, W.T., et al., 2002. Cells in human postmortem brain tissue slices remain alive for several weeks in culture. FASEB J. 16 (1), 54-60.

Viola, K.L., Klein, W.L., 2015. Amyloid beta oligomers in Alzheimer's disease pathogenesis, treatment, and diagnosis. Acta Neuropathol. 129 (2), 183-206.

Wallach, G., Lallouette, J., et al., 2014. Glutamate mediated astrocytic filtering of neuronal activity. PLoS Comput. Biol. 10 (12), e1003964.

Walsh, D.M., Teplow, D.B., 2012. Alzheimer's disease and the amyloid beta-protein. Prog. Mol. Biol Transl. Sci. 107, 101-124.

Wang, L., Peng, S.Y., et al., 2010. Effects and mechanism of aromatic aminoketone SY0916 on osteoclastic bone destruction. Acta Pharmacol. Sin. 31 (4), 470-475. 